\title{
Image fusion of coronary CT angiography and cardiac perfusion MRI A pilot study
}

\section{Journal Article}

\section{Author(s):}

Stolzmann, Paul; Alkadhi, Hatem; Scheffel, Hans; Hennemuth, Anja; Kuehnel, Caroline; Baumueller, Stephan; Kozerke, Sebastian; Falk, Volkmar; Marincek, Borut; Donati, Olivio F.

Publication date:

2010-05

Permanent link:

https://doi.org/10.3929/ethz-b-000027129

Rights / license:

In Copyright - Non-Commercial Use Permitted

Originally published in:

European Radiology 20(5), https://doi.org/10.1007/s00330-010-1746-2 
Paul Stolzmann

Hatem Alkadhi

Hans Scheffel

Anja Hennemuth

Caroline Kuehnel

Stephan Baumueller

Sebastian Kozerke

Volkmar Falk

Borut Marincek

Olivio F. Donati

\section{Image fusion of coronary CT angiography and cardiac perfusion MRl: a pilot study}

Received: 11 September 2009

Revised: 11 December 2009

Accepted: 24 January 2010

Published online: 4 March 2010

(C) European Society of Radiology 2010
P. Stolzmann · H. Alkadhi ·

H. Scheffel - S. Baumueller .

B. Marincek · O. F. Donati

Institute of Diagnostic Radiology,

University Hospital Zurich,

Zurich, Switzerland

H. Alkadhi $(\square)$

Cardiac MR PET CT Program,

Massachusetts General Hospital,

165 Cambridge Street, Suite 400,

Boston, MA, 02114, USA

e-mail: halkadhi@partners.org

A. Hennemuth · C. Kuehnel

Fraunhofer MEVIS,

Bremen, Germany

\section{S. Kozerke}

Institute for Biomedical Engineering,

University and ETH Zurich,

Zurich, Switzerland

V. Falk

Clinic for Cardiovascular Surgery,

University Hospital Zurich,

Zurich, Switzerland

\begin{abstract}
Objective: To develop a tool for the image fusion of computed tomography coronary angiography (CTCA) and cardiac magnetic resonance imaging (CMR). Methods: Surface representations and volume-rendered images from fused CTCA/CMR data of five patients with significant coronary artery disease (CAD) on CTCA and perfusion deficits on CMR were generated using a newly developed software prototype. The spatial relationship of significant coronary artery stenosis at CTCA and myocardial defects at CMR was evaluated. Results: Registration of CTCA and CMR images was possible
\end{abstract}

in all patients. The comprehensive three-dimensional visualisation of fused CTCA and CMR data accurately demonstrated the relationship between coronary artery stenoses and myocardial defects in all patients.

Conclusion: The introduced tool enables image fusion of CTCA and CMR data sets and allows for correct superposition of the coronary arteries derived from CTCA onto the corresponding myocardial segments derived from CMR. The method facilitates the comprehensive assessment of the functionally relevant $\mathrm{CAD}$ by the exact allocation of culprit coronary stenoses to corresponding myocardial defects at a low radiation dose.

Keywords Computed tomography · Integrative imaging $\cdot$ Myocardial perfusion $\cdot$ Magnetic resonance imaging · Image fusion · Image registration

\section{Introduction}

The precise assessment of haemodynamically relevant coronary artery disease (CAD) requires both anatomic information on the coronary arteries as well as functional information on myocardial perfusion and viability [1].

Prospectively electrocardiography (ECG)-triggered computed tomography coronary angiography (CTCA) is a non-invasive tool for the visualisation of coronary artery anatomy associated with a low radiation dose while maintaining diagnostic performance compared with cath- eter angiography (CA) [2, 3]. Myocardial perfusion and viability can be evaluated using cardiac magnetic resonance imaging (CMR) with reported accuracies similar to those of nuclear tests, but with the advantages of higher spatial resolution and no associated ionising radiation [4].

Evaluating anatomic and functional information side by side, as done in the simultaneous consideration of CTCA and CMR images, is known to cause difficulties in the correct allocation of vascular territories to myocardial segments [5]. In $17-50 \%$ of cases, standard myocardial distribution territories according to standard definitions [6] 
do not correspond with actual coronary anatomy $[5,7]$. A solution may be achieved by integrating both CTCA and CMR into a single dataset by image fusion. This noninvasive imaging approach should allow for the direct designation of ischaemic or infarcted myocardium to its subtending coronary stenoses, along with a very low radiation burden to the patient.

The purpose of this study was to develop a method for the image fusion of CTCA and CMR data, to demonstrate its feasibility, and to test this method in a small series of consecutive patients.

\section{Materials and methods}

\section{Patients}

Five consecutive patients (two women; age, $63 \pm 12$ years; range, 52-77) with significant $\mathrm{CAD}$ (i.e. significant coronary stenosis) on CTCA and perfusion deficits on CMR were enrolled in this study $[8,9]$. All patients underwent CTCA and CMR on the same day. CA was performed within $8 \pm 5$ days of the non-invasive tests which confirmed the significant coronary stenoses as detected by CTCA in all patients.

The institutional review board approved the study and all participants gave written informed consent.

\section{Data acquisition}

CTCA data were acquired on a dual-source CT system (Somatom Definition, Siemens Healthcare, Forchheim, Germany) using a prospectively ECG-triggered protocol. All patients received a single $2.5-\mathrm{mg}$ dose of sublingual isosorbide dinitrate. Seventy to $80 \mathrm{ml}$ of contrast medium (iopromide, Ultravist 370, Bayer Schering Pharma, Berlin, Germany) was administered at a flow rate of $5-6 \mathrm{ml} / \mathrm{s}$, followed by an isovolumetric second phase consisting of a $20 \%$ contrast agent, $80 \%$ saline solution mixture. All patients were in sinus rhythm during data acquisition. Data were acquired in the cranio-caudal direction during midinspiration using the following parameters: detector collimation, $2 \times 32 \times 0.6 \mathrm{~mm}$; slice acquisition, $2 \times 64 \times 0.6 \mathrm{~mm}$ by means of a z-flying spot; gantry rotation time, $0.33 \mathrm{~s}$. Attenuation-based tube current modulation was used with a reference tube current-time product of $190 \mathrm{mAs}$ per rotation. The data acquisition window was set at $70 \%$ of the R-R interval; the temporal resolution was $83 \mathrm{~ms}$. CTCA images were reconstructed in a mono-segmental reconstruction algorithm with a slice thickness of $0.6 \mathrm{~mm}$ (increment, $0.4 \mathrm{~mm}$ ) and using a medium smooth-tissue convolution kernel (B26 f). Low-dose CTCA was successfully performed in all patients without complications. The average effective radiation dose per patient was $2.2 \pm 0.6 \mathrm{mSv}$.

Cardiac magnetic resonance imaging was performed with 1.5-T clinical magnetic resonance imaging (MRI)
(Achieva, Philips Medical Systems, Best, The Netherlands). Dedicated cardiac phased-array receiver coils were used for signal reception (five elements). Four representative short-axis sections were obtained. Pharmacological stress was applied using adenosine administered intravenously at $140 \mu \mathrm{g}$ per kilogram of body weight over $3 \mathrm{~min}$. Acquisition of perfusion-CMR images was started immediately after the injection of gadobutrol (Gadovist 1,0; Bayer $\mathrm{AG}$ ) at $0.1 \mathrm{mmol}$ per kilogram of body weight at an injection rate of $5 \mathrm{ml} / \mathrm{s}$, followed by a $40 \mathrm{ml}$ saline flush. Ten minutes after stress perfusion imaging, a second bolus of $0.1 \mathrm{mmol}$ gadobutrol was injected and rest perfusion images were obtained. K-t sensitivity encoding perfusionCMR imaging was used in combination with a saturation recovery gradient-echo pulse sequence for both of these sequences (repetition time/echo time, 3.1/1.1 ms; flip angle, $20^{\circ}$; saturation pre-pulse delay, $110 \mathrm{~ms}$; partial Fourier sampling; acquisition window, $120 \mathrm{~ms}$; section thickness, $10 \mathrm{~mm}$; k-t factor of 5, with $11 \mathrm{k}$-t interleaved training profiles; effective acceleration, 3.7 ; three sections acquired sequentially during a single R-R interval). Highspatial resolution perfusion-CMR images were acquired with an in-plane resolution of $2.00 \times 2.00 \mathrm{~mm}^{2}$ (reconstructed $1.25 \times 1.25 \mathrm{~mm}^{2}$ ).

Ten minutes after rest perfusion, late gadoliniumenhanced images (LGE) were acquired in a continuous short-axis view using an inversion-recovery gradientrecalled echo CMR sequence with the following parameters: field of view, $350-400 \mathrm{~mm}$; repetition time/echo time, 7.4/4.3 ms; inversion time, $200-350 \mathrm{~ms}$; flip angle, $20^{\circ}$; matrix, $240 \times 240$; slice thickness, $10 \mathrm{~mm}$. The optimal inversion time was chosen to null the signal from normal myocardium.

CMR data acquisition could be successfully performed in all patients without complications.

\section{Data post-processing}

Images from prospectively ECG-triggered CTCA and CMR were analysed using software developed by Fraunhofer MEVIS (Bremen, Germany) based on the MeVisLab SDK. The software imports DICOM data from CT- and MR-scanners from most vendors. Analysis results were derived from two advanced software assistants, processing CTCA on the one hand, and perfusion and LGE images on the other hand.

First, the images from CTCA and the LGE images from CMR were transformed into a common coordinate system. Image studies were aligned using a rigid transformation with respect to three characteristic user-defined landmarks, which were defined in both CTCA and LGE images (e.g. apex, base and septum). Additional manual correction was supported, and the resulting transformation was used to change the image position and orientation defined in the DICOM header. Thereby, image intensities were not 
affected. With the first software assistant, the coronary tree on prospectively ECG-triggered CTCA was semi-automatically segmented using an advanced progressive region-growing algorithm [10]. Using the second software assistant, CMR data were analysed performing a perfusion analysis with the affine and B-spline registration methods for motion correction. Various perfusion parameters were determined, e.g. time to peak, upslope, area under curve and peak enhancement [11]. For our analysis, semiquantitative parameters from the voxel-intensity curves of the perfusion sequence and semi-automatic segmentation in the resulting parameter images were used. The perfusion analysis started with an interactive segmentation of the myocardium. Thanks to the initial motion correction step, only one segmentation had to be carried out per slice. Before computation of the upslope parameter, the voxel intensity curves were corrected by their baseline value in the reference time frame. A segmentation method for the stress perfusion sequence and a subsequent region-based calculation of the myocardial perfusion reserve to control the result were performed. To segment the hypoperfused regions, a segmentation threshold was defined in the histogram of the computed slope parameter image through fitting a mixture model. The threshold was defined as the intersection of two curves, which represent the parameter distributions in hypoperfused and healthy tissue.

For LGE analysis, the first step of late enhancement detection consisted of the segmentation of the myocardium. This was done semi-interactively using a Live-Wire algorithm. Once the myocardium was segmented, the intensity distribution was analysed by fitting a mixture model of a Rayleigh distribution and a Gaussian distribution. These distributions are considered adequate for representing low and high intensities, respectively. The scar tissue portion was determined by fitting a mixture model to the histogram of the myocardial region [11]. Hence, not only the portion of infarcted tissue in a predefined tissue region was measured but also the surface of the infarcted region was located. This information was then used to visualise location, shape and transmurality of the infarction.

For visualisation of the data, result masks of the left ventricle (LV) were colour-coded with perfusion and LGE parameters. Next, these were overlaid on a surface representation of the ventricle. Then, in order to demonstrate the relation between the coronary arteries segmented from CTCA and the myocardial defects from CMR, registered surfaces and volume renderings of the coronary arteries and LV were integrated with parameters from CMR. In addition, the segmented coronary arteries from CTCA and the information from perfusion-CMR and LGE-CMR were integrated into a volume rendering based on the CTCA data. In summary, two visualisation modes are possible:

1. An integrated view of surface renderings of the segmented coronary tree from CTCA with the LV overlaid with segmented myocardial defects (e.g. LGE or perfusion deficits) from CMR. The mask representing the LV is colour-coded with the distance from the surface to the infarction and thus shows transmurality.

2. An integrated view of the segmented myocardial defects from CMR with a volume rendering based on CTCA data.

The relation between the perfusion or LGE defects and a significant coronary artery showing stenosis was evaluated visually and classified as match or mismatch in consensus by two radiologists. No quantitative assessment of registration accuracy was performed.

\section{Results}

There was no problematic registration of CTCA and CMR images. The comprehensive three-dimensional visualisation of volume-rendered images fused with surface representations of hypo-perfused or scar areas accurately demonstrated the relationship between coronary artery stenoses and perfusion deficits or LGE in all five patients.

Table 1 Patient data ( $M$ male, $F$ female, $A C P$ atypical chest pain, $L A D$ left anterior descending coronary artery, $L C X$ left circumflex coronary artery, $R C A$ right coronary artery, $D 1$ first diagonal branch of the left anterior descending coronary artery)

\begin{tabular}{lllllll}
\hline Patient no. Gender & Age (years) & Symptoms & Low-dose CTCA & CMR & Fused \\
CTCA/CMR \\
Match/mismatch
\end{tabular}


Coronary stenosis matched the corresponding perfusion deficit or LGE in all patients (Table 1).

As an illustrative case, we present the imaging findings of a 52-year-old male patient with two-vessel disease (Table 1, case 2). CTCA demonstrated occlusion of the proximal RCA and stenosis of the first diagonal branch (D1) (Fig. 1a, b). CMR detected a myocardial scar on LGE images in the mid-inferior and basal inferior myocardial segments (Fig. 1c) with corresponding perfusion deficits in this area of infarction. An additional perfusion deficit was located in the basal anterior/anteroseptal segments (Fig. 1d).

Using fused coronary arteries segmented from prospectively ECG-triggered CTCA and a surface representation of the LV with LGE (Fig. 1e) and perfusion-CMR (Fig. 1f), areas of infarction and perfusion deficits were allocated to the corresponding culprit coronary lesions. Similarly, integrating the segmented coronary arteries from prospectively ECGtriggered CTCA and the information from perfusion-CMR
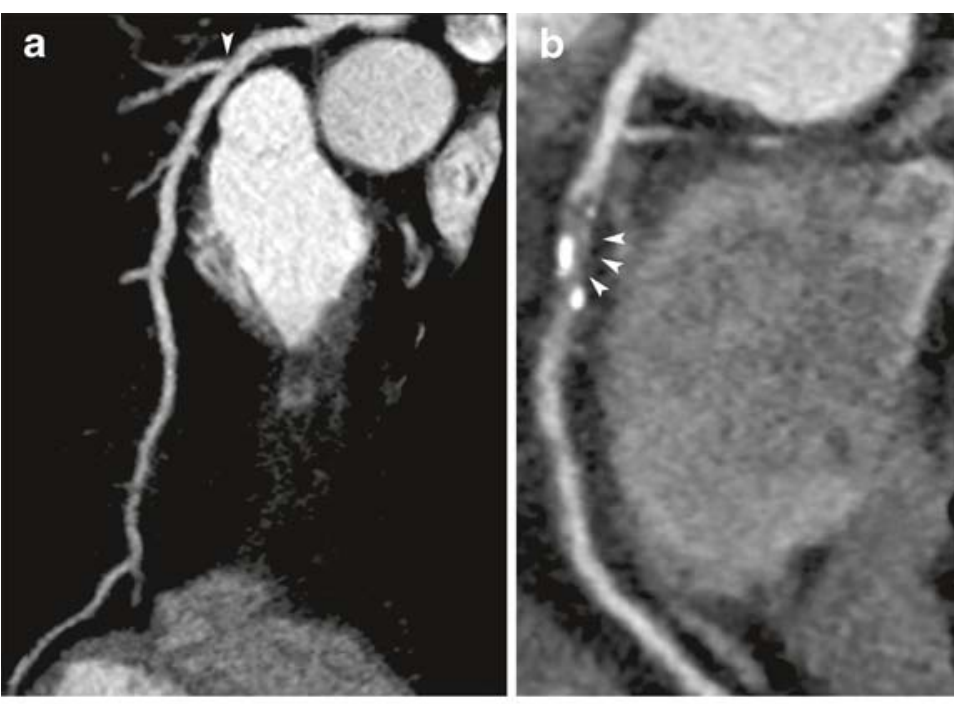

e
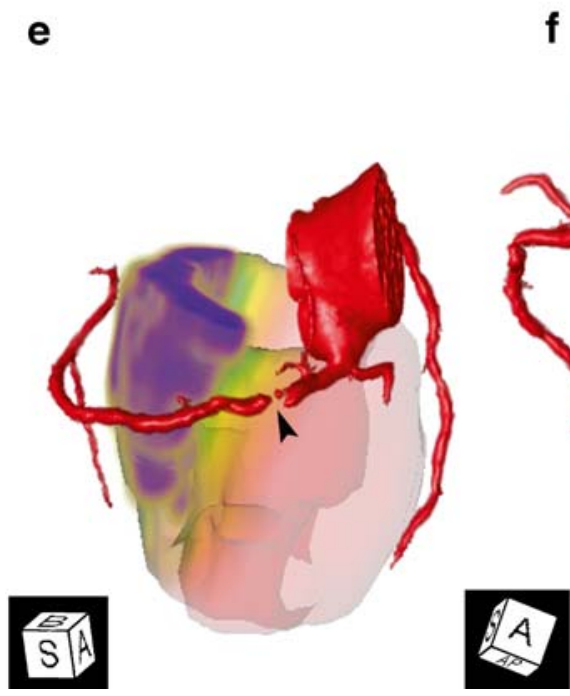

f

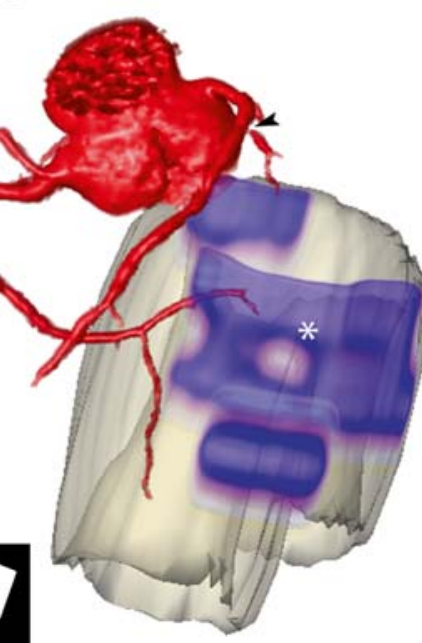

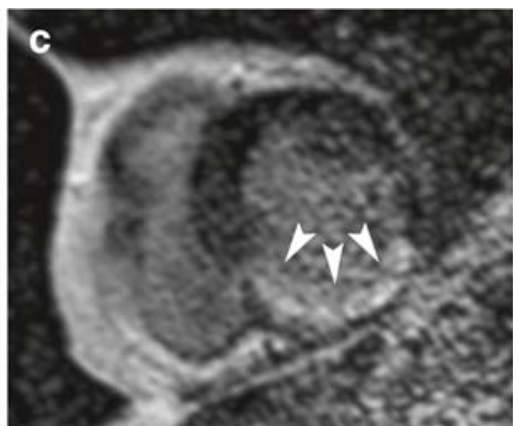

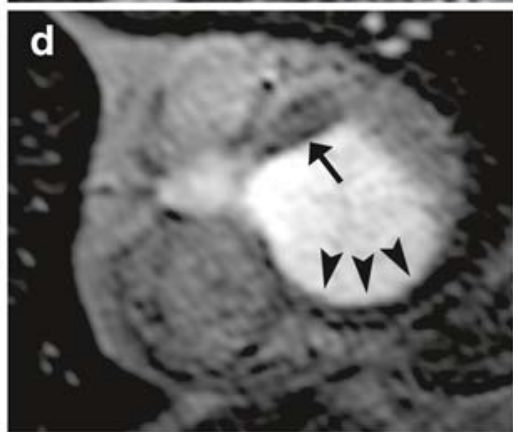

g

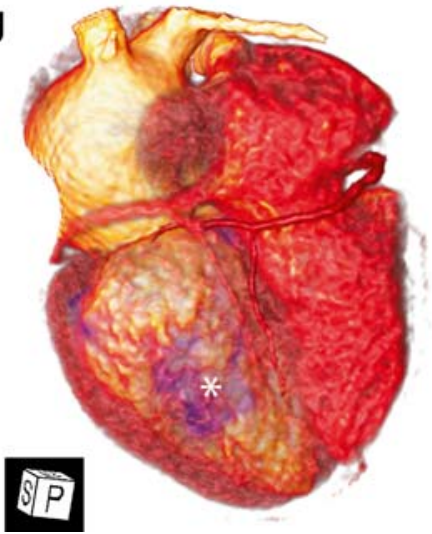

Fig. 1 A 52-year-old male patient with CAD. Prospectively ECGtriggered CTCA shows stenosis of the first diagonal branch (D1) (arrowhead in a) and occlusion of the proximal right coronary artery (RCA, arrowheads in b). CMR depicts LGE in inferior segments (arrowheads in c showing mid inferior segment). Adenosine stress perfusion-CMR reveals perfusion deficits in the area of myocardial infarction (arrowheads in d) and in the basal anterior/anteroseptal segment (arrow in d). Surface-rendered representation of the left ventricle with LGE data integrated with the segmented coronary arteries (e) shows the relationship between the RCA occlusion (arrowhead) and the corresponding inferior myocardial infarction (purple area). The percentage of transmurality is displayed in a colour-coded fashion. Surface representation of perfusion deficits and volume-rendered coronary arteries (f) demonstrate stenosis of D1 (arrowhead) with corresponding perfusion deficit in the basal anterior/anteroseptal segments (purple area). Note the additional perfusion deficit matching the area of infarction (asterisks). In a volume-rendered representation using the CTCA data complemented by CMR information (g), the area of myocardial infarction (asterisks) can easily be attributed to the occlusion of the RCA. Projection angles in images e-g: $A$ anterior, $S$ septal, $B$ basal, $A P$ apical, $P$ posterior 
into a volume rendering based on the CTCA data, the area of inferior myocardial infarction was precisely allocated to the occlusion in the right coronary artery (Fig. 1g).

\section{Discussion}

In this pilot study, image fusion of prospectively ECGtriggered CTCA and CMR was possible in all five consecutive patients without misregistration problems. The relationship between the coronary artery stenoses and corresponding myocardial defects could be accurately determined in all fused datasets. Our method provides, at a low radiation dose, an easily interpretable view of the heart, integrating high resolution, anatomic information of the coronary arteries with precise functional information.

The definition of functionally relevant coronary stenoses solely based on anatomic criteria remains controversial. Although it is widely accepted that a coronary stenosis causing luminal narrowing $>50 \%$ is "significant", other additional factors that cannot fully be evaluated by the depiction of anatomy alone determine whether or not a given lesion causes ischaemia. Therefore, proof of ischaemia is recommended before revascularisation procedures [1]. Integration of anatomic and functional information from CTCA and CMR has been performed mentally so far. As there are numerous various anatomical variants of coronary arteries, a side-by-side interpretation of CTCA and CMR studies may render integration difficult. This may lead to inaccurate allocation of the culprit coronary stenosis to its subtended myocardial territory.

Image fusion for the integrative evaluation of functionally relevant $\mathrm{CAD}$ has been tested using single-photon emission CT or positron emission tomography $[7,12]$. There is only one study so far reporting on the image fusion of four- and 16-detector-row CTCA with LGE data obtained from CMR [5]. In this study, however, semi-automatically delineated coronary arteries were superimposed on only two-dimensional CMR slices. Our approach, however, allowed the integration of the segmented coronary arteries from CTCA with perfusion and LGE images from CMR in a threedimensional fashion without misregistration in all patients. Hence, our study can be considered one of the first to introduce a new and precise, semiautomatic three-dimensional image fusion tool for CTCA and CMR data considering not only viability information from LGE images but also information about myocardial perfusion from CMR. The anatomic information from the segmented coronary arteries from CTCA is hereby integrated with perfusion as well as LGE information from CMR image data. Particularly intriguing is the low effective radiation dose of approximately $1-3 \mathrm{mSv}$ associated with this non-invasive imaging approach [13], including both the evaluation of coronary anatomy by prospectively ECG-triggered CTCA and myocardial function by CMR.

In conclusion, our early results indicate that image fusion of prospectively ECG-triggered CTCA and CMR datasets is feasible and allows for a correct superposition of the coronary arteries derived from CTCA onto the corresponding myocardial segments derived from CMR. Our method thus allows for a comprehensive assessment of functionally relevant CAD at a low radiation dose. Further studies are required to evaluate both the potential clinical benefit of CTCA/CMR image fusion compared with side-by-side analysis and its impact on clinical decision-making.

Acknowledgements This study was supported by the National Center of Competence in Research, Computer Aided and Image Guided Medical Interventions of the Swiss National Science Foundation.

\section{References}

1. Smith SC Jr, Feldman TE, Hirshfeld JW Jr et al (2006) ACC/AHA/SCAI 2005 guideline update for percutaneous coronary intervention: a report of the American College of Cardiology/ American Heart Association Task Force on Practice Guidelines (ACC/ AHA/SCAI Writing Committee to Update the 2001 Guidelines for Percutaneous Coronary Intervention). J Am Coll Cardiol 47:e1-121. doi:10.1016/j. jacc.2005.12.001
2. Scheffel H, Alkadhi H, Leschka S et al (2008) Low-dose CT coronary angiography in the step-and-shoot mode: diagnostic performance. Heart 94:11321137. doi:10.1136/hrt.2008.149971

3. Stolzmann P, Leschka S, Scheffel H et al (2008) Dual-source CT in step-andshoot mode: noninvasive coronary angiography with low radiation dose. Radiology 249:71-80. doi:10.1148/radiol.2483072032

4. Schwitter J, Wacker CM, van Rossum AC et al (2008) MR-IMPACT: comparison of perfusion-cardiac magnetic resonance with single-photon emission computed tomography for the detection of coronary artery disease in a multicentre, multivendor, randomized trial. Eur Heart J 29:480-489. doi:10.1093/eurheartj/ ehm617
5. Setser RM, O'Donnell TP, Smedira NG et al (2005) Coregistered MR imaging myocardial viability maps and multidetector row CT coronary angiography displays for surgical revascularization planning: initial experience. Radiology 237:465-473. doi:10.1148/ radiol.2372040236 
6. Cerqueira MD, Weissman NJ, Dilsizian V et al (2002) Standardized myocardial segmentation and nomenclature for tomographic imaging of the heart: a statement for healthcare professionals from the Cardiac Imaging Committee of the Council on Clinical Cardiology of the American Heart Association. Circulation 105:539-542
7. Schindler TH, Magosaki N, Jeserich M et al (1999) Fusion imaging: combined visualization of 3D reconstructed coronary artery tree and 3D myocardial scintigraphic image in coronary artery disease. Int J Card Imaging 15:357-368 discussion 369-370

8. Scheffel H, Stolzmann P, Alkadhi H et al (2010) Low-dose CT and cardiac MR for the diagnosis of coronary artery disease: accuracy of single and combined approaches. Int J Cardiovasc Imaging. [Epub ahead of print]

9. Stolzmann P, Donati OF, Scheffel H et al (2010) Low-dose CT coronary angiography for the prediction of myocardial ischaemia. Eur Radiol 20(1):56-64

10. Bock S, Kuhnel C, Boskamp T, Peitgen H-O (2008) Robust vessel segmentation. In: Giger ML, Karssemeijer N (eds) SPIE, p 691539
11. Hennemuth A, Seeger A, Friman O et al (2008) A comprehensive approach to the analysis of contrast enhanced cardiac MR images. IEEE Trans Med Imaging 27:1592-1610. doi:10.1109/ TMI.2008.2006512

12. Nishimura Y, Fukuchi K, Katafuchi T et al (2004) Superimposed display of coronary artery on gated myocardial perfusion scintigraphy. J Nucl Med 45:1444-1449. doi:45/9/1444 [pii]

13. Alkadhi H (2009) Radiation dose of cardiac CT-what is the evidence? Eur Radiol 19:1311-1315 